\title{
Long distance fiber Bragg grating strain sensor interrogation using high speed Raman-based Fourier domain mode-locked fiber laser with recycled residual Raman pump
}

\author{
Sunduck Kim, Oh-Jang kwon, and Young-Geun Han \\ Department of Physics, Hanyang University \\ 17 Haengdang-dong, Seongdong-gu, Seoul 133-791, Korea \\ TEL: +82-2-2220-2551, FAX: +82-2-2295-6868, E-mail: yghan@hanyang.ac.kr
}

\begin{abstract}
We propose a novel fiber Bragg grating (FBG) sensor interrogation system using a Raman-based Fourier domain mode locking (FDML) fiber laser for a high speed and a long distance measurement. To improve transmission efficiency of the sensing probe signal over a long distance of $20-\mathrm{km}$, the residual pump powers are recycled. The external strain change can be measured by detecting the tine interval between two reflected signals from two FBGs as the Rama-based FDML is swept. The measured strain sensitivities with respect to the time are $0.19 \mathrm{~ns} / \mu$ strain.
\end{abstract}

Keywords: Fiber Bragg grating sensor, Raman amplification, Fourier domain mode-locked fiber laser, Strain measurement

\section{INTRODUCTION}

Fiber Bragg gratings (FBGs) have attracted considerable attention in versatile sensing applications because of their advantages, such as compactness, fiber compatibility, immunity to electromagnetic interference, and multipoint sensing capability [1]. The FBG can be implemented to measure physical quantities including strain, temperature, pressure, and vibration by detecting a shift in the reflection center wavelength [2]. Since the interrogation technique of the FBG-based sensing system should provide fast monitoring speed, high signal power, high accuracy, and sensitivity, there have been various researches on the detection techniques based on passive optical filters and interferometers [3, 4]. For the multiple FBG sensor interrogation technique with high speed and sensitivity, wavelength-swept fiber lasers over the spectral range of FBG sensors have been proposed as a desirable optical source $[5,6]$. When the laser is swept over the spectral range of the FBG sensors, signals reflected from FBGs are simultaneously detected by a single detector, which provides improved performance compared with the conventional passive interrogation method. However, the maximum speed of the swept rate was physically limited to a few $\mathrm{kHz}$ due to the filter function, the amplified stimulated emission (ASE) intensity, the saturation power, the laser gain, and cavity roundtrip time [7]. Recently, Fourier domain mode locking (FDML) fiber laser based on a semiconductor optical amplifier (SOA) has been developed to overcome swept speed by modulating the optical filter synchronously with the round trip time of the laser cavity and applied to FBG sensor interrogation $[8,9]$. However, the maximum transmission distance in FBG sensor systems is limited mainly due to the signal loss after a long distance of a remote sensing FBG. For long distance measurement, an FBG sensor system using spectrum-limited FDML laser based on Raman amplification was introduced [10]. While the measurement distance was increased by extending the laser cavity length, the swept rate became low due to the long cavity length, and the driving frequency for the swept laser had to be adjusted for reading the signal from each FBG sensor installed at different distances. Therefore, it is necessary to develop a new and simple interrogation technique with high swept rate at a long distance of $20 \mathrm{~km}$. In this paper, a novel FBG strain sensor interrogation using Raman-based FDML fiber laser is presented for high speed and long distance measurement. After a pump powers of $717 \mathrm{~mW}$ passes through a Raman gain fiber, the residual pump powers of $205 \mathrm{~mW}$ are recycled to improve the optical power of sensing signal for remote sensing at a long distance over $20-\mathrm{km}$. The swept range and rate of the Raman-based FDML fiber laser are $37.2 \mathrm{~nm}, 30.8$ $\mathrm{kHz}$, respectively. The measured strain sensitivities with respect to the wavelength and the time are $0.81 \mathrm{pm} / \mu$ strain and $0.19 \mathrm{~ns} / \mu$ strain, respectively. Using the proposed scheme, we can obtain typical response from the FBG sensor interrogation as a function of strain.

OFS2012 22nd International Conference on Optical Fiber Sensors, edited by

Yanbiao Liao, Wei Jin, David D. Sampson, Ryozo Yamauchi, Youngjoo Chung, Kentaro Nakamura, Yunjiang Rao,

Proc. of SPIE Vol. 8421, 84213U · (c) 2012 SPIE · CCC code: 0277-786/12/\$18 · doi: 10.1117/12.966814 


\section{EXPERIMENTS AND RESULTS}

Figure 1(a) shows a schematic diagram of the experiment setup of an FBG sensor interrogation system using Ramanbased FDML fiber laser with recycled residual Raman pump. The Raman-based FDML fiber laser consists of two isolators for a unidirectional sweeping, a 2-km highly nonlinear fiber (HNLF) as a Raman gain medium, a $1.5-\mathrm{km}$ dispersion compensating fiber (DCF), a 3-km single mode fiber (SMF), a polarization controller (PC), a fiber FabryPerot tunable filter (FFP-TF), and a function generator. The Raman pump laser with a power of 717-mW was launched into the HNLF to induce Raman amplification. The FDML can be readily realized if the FFP-TF is periodically driven with a frequency equal to optical round-trip time within the laser cavity [11]. Light from one frequency sweep passes through the FFP-TF, propagates through the cavity, and returns to the FFP-TF at exactly the time when the FFP-TF is again tuned to the same frequency. An entire swept wavelength is optically stored within a long laser cavity and FDML lasers produce a train of frequency sweep that have spectral information in time domain [11]. Since the chromatic dispersion severely induces synchronization errors, the the DCF and the SMF should be exploited to manage the dispersion within the FDML cavity [12]. The output of the Raman-based FDML fiber laser was launched into two FBGs after passing through the $20-\mathrm{km}$ SMF. The residual Raman pump power of $205 \mathrm{~mW}$ was injected into the $20-\mathrm{km}$ SMF to induce the additional Raman gain to transmit the proposed Raman-based FDML signal to a long distance over 20-km. FBG1 and FBG2 with central wavelength of 1548.1 and $1549.9 \mathrm{~nm}$ and reflectivities of 97.2 and $94.1 \%$ were used as a reference and a sensing probe, respectively. The reflected signals from two FBGs are detected by an optical spectrum analyzer (OSA) and a photo-detector (PD) connected to an oscilloscope. The oscilloscope is triggered at the driving frequency of the FFP-TF to record pulse data in the same time span.

Raman-based FDML fiber laser

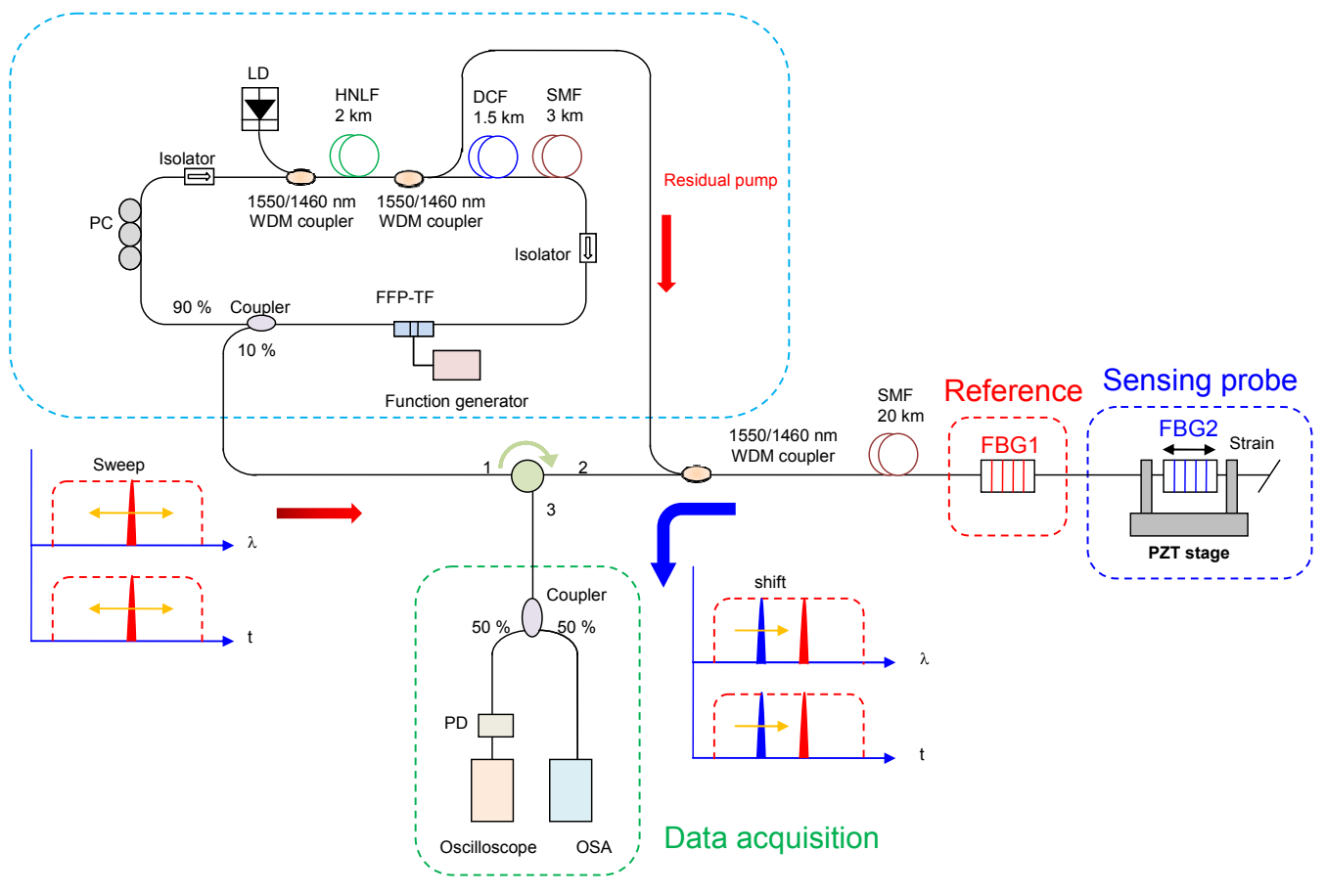

Figure 1. Experimental setup for the proposed FBG sensor using the Raman-based FDML fiber laser with the recycling residual Raman pump.

When the Raman-based FDML fiber laser is repeatedly swept over the spectral range of two FBGs, the signals reflected from the FBGs, which consists of a series of pulses in the time domain, are detected at the PD. The center wavelength of the FBG2 as a sensing probe is changed by strain, resulting in the variation of the wavelength spacing between two FBGs. The time intervals detected in the PD corresponding to the wavelength spacing between two FBGs must be changed. Consequently, the external strain change can be measured by acquiring the time intervals between two reflected signals from two FBGs as the proposed Raman-based FDML is swept.

Figure 2(a) shows the output spectrum of the Raman-based FDML fiber laser measured by the OSA. The operating 
frequency of the FFP-TF was set to be $30.8 \mathrm{kHz}$ with respect to the total length of $6.5 \mathrm{~km}$ in the cavity. At the peak hold mode of the OSA, the full width of half maximum (FWHM) and the extinction ratio were measured to be $37.2 \mathrm{~nm}$ centered at $1547 \mathrm{~nm}$ and $55.1 \mathrm{~dB}$, respectively. The temporal transient intensity profiles of the Raman-based FDML fiber laser, which was measured by the photo-detector and the oscilloscope as shown in Fig. 2(b). In comparison with the FDML based on a SOA gain medium, the intensity profile exhibits higher fluctuations. However, since the proposed FBG sensor system reads the variation of the pulse interval between a reference FBG and a sensing FBG, the fluctuation of the output profile can be ignored.
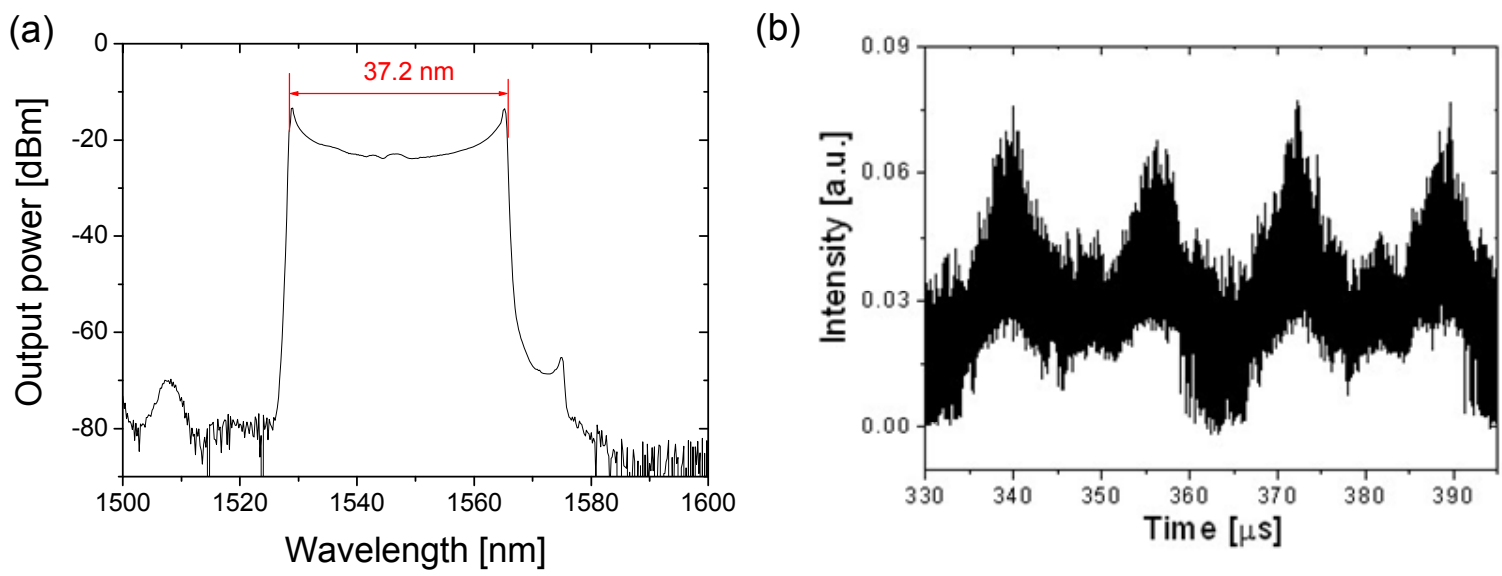

Figure 2. (a) Output spectrum and (b) temporal transient intensity profile of the Raman-based FDML fiber laser.

Figure 3 shows output spectra of the proposed Raman-based FDML laser in the time domain with variations in strain, which is measured by using a PD and an oscilloscope. Since strain is applied to the FBG2 as the sensing probe, the wavelength spacing between two FBGs is reduced by strain. Therefore, the time interval between two reflected signals from two FBGs measured by using the PD should be reduced. When the strain in a range from 0 to $380 \mu$ strain was applied to the FBG2 by using a piezoelectric transducer (PZT), the center wavelength of the FBG2 will be shifted to longer wavelengths and eventually the time interval should be hanged. The inset in the Fig 3 shows the signals of the FBG1 and FBG2 after a 40-km round trip distance in the time domain. Due to the residual Raman pump into the 20-km SMF, sufficient intensities of the reflected signal from FBG1 and FBG2 can be obtained to measure the variation of the remote strain, which is difficult to implement with a conventional FDML based on a SOA gain medium. Figure 4 depicts the variation of the wavelength spacing and the time interval as a function of strain. The strain sensitivity is estimated to be $0.19 \mathrm{~ns} / \mu$ strain.

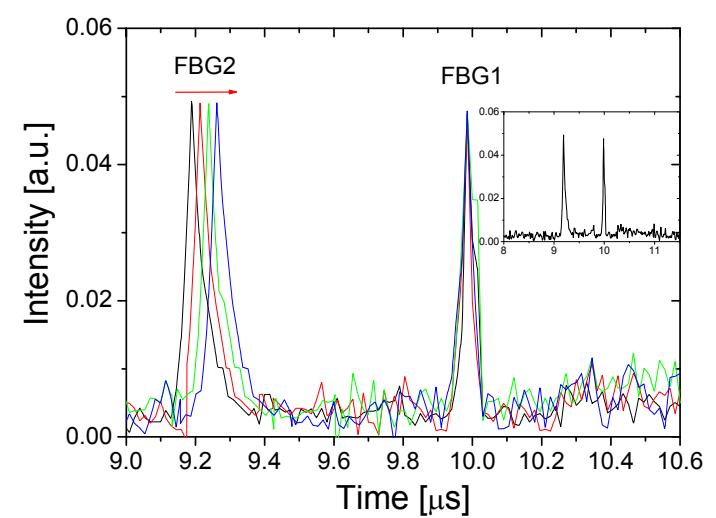

Figure 3. Measured optical output with variation in strain

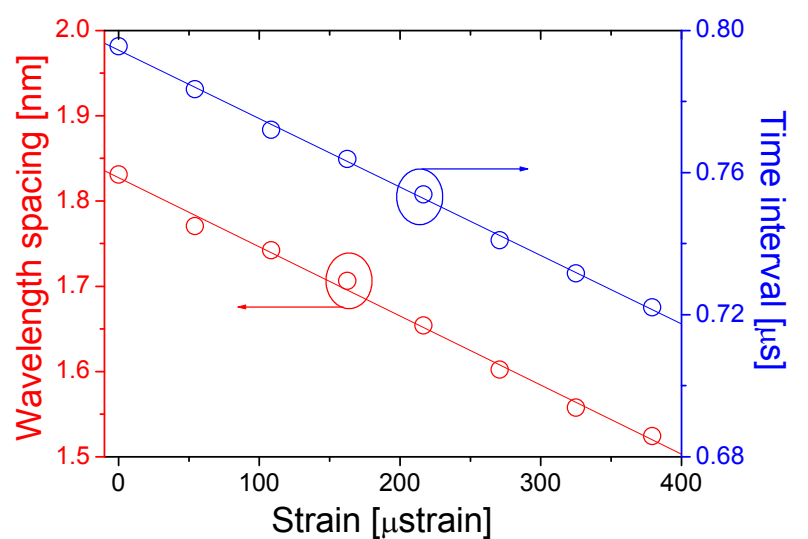

Figure 4. Wavelength spacing and time interval as a function of strain 


\section{CONCLUSION}

A long distance FBG sensor interrogation system using a high-speed Raman-based FDML fiber laser has been demonstrated. In the proposed FBG sensor system, the residual pump power can be recycled to reduce the transmission loss with a 20-km distance. The Raman-based FDML fiber laser showed a high performance at a $30.8 \mathrm{kHz}$ sweeping rate and a wavelength scan range of $37.2 \mathrm{~nm}$. Since the swept rate is determined by the round trip time in the cavity, it is possible to increase the swept rate up to hundreds $\mathrm{kHz}$ by reducing the cavity length. By monitoring the variation of the time interval between two reflected signals from two FBGs, we can effectively measure the external strain change. The proposed Raman-based FDML fiber laser can be useful for high-speed and long distance FBG interrogation systems.

\section{REFERENCES}

[1] A. D. Kersey, M. A. Davis, H. J. Patrick, M. LeBlanc, K. P. Koo, C. G. Askins, M. A. Putnam and E. J. Friebele, "Fiber grating sensors," J. Lightwave Technol., 15, 1442 - 1463 , 1997.

[2] B. Lee, "Review of the present status of optical fiber sensors," Opt. Fiber Technol., 9, 57 - 79, 2003.

[3] S. M. Melle, K. Liu, and R. M. Measures, "A Passive Wavelength Demodulation System for Guided-Wave Bragg Grating Sensors," IEEE Photonic. Tech. L., 4, 516 - 518, 1992.

[4] A. D. Kersey, T. A. Berkoff, and W. W. Morey, "High-resolution fibre-grating based strain sensor with interferometric wavelength-shift detection," Electron. Lett., 28, 236 - 238, 1992.

[5] S. H. Yun, D. J. Richardson, and B. Y. Kim, "Characterization Interrogation of fiber grating sensor arrays with a wavelength-swept fiber laser," Opt. Lett., 23, 843 - 845, 1998.

[6] S. Y. Ryu and C. S. Hong, "Development of fiber Bragg grating sensor system using wavelength-swept fiber laser," Smart Mater. Struct., 11, $468-473,2002$.

[7] R. Huber, M. Wojtkowski, K. Taira, J. Fujimoto, and K. Hsu, "Amplified, frequency swept lasers for frequency domain reflectometry and OCT imaging:design and scaling principles," Opt. Express, 13, 3513 - 3528, 2005.

[8] E. J. Jung, C. S. Kim, M. Y. Jeong, M. K. Kim, M. Y. Jeong, W. Jung, and Z. Chen, "Characterization of FBG sensor interrogation based on a FDML wavelength swept laser," Opt. Express, 16, 16552 - 16560, 2008.

[9] B. C. Lee, E. J. Jung, C. S. Kim, and M. Y. Jeong, "Dynamic and static strain fiber Bragg grating sensor interrogation with a $1.3 \mathrm{~mm}$ Fourier domain mode-locked wavelength-swept laser," Meas. Sci. Techno., 21, 094008, 2010.

[10] J. J. Fu, W. S. Liu, D. Chen, and S. He, "Ultra-long-distance FBG sensor system based on spectrum-limited Fourier domain modelocking fibre laser with Raman pumps," Electron. Lett., 44, 961 - 963, 2008.

[11] R. Huber, M. Wojtkowski, and J. G. Fujimoto, "Fourier Domain Mode Locking (FDML): A new laser operating regime and applications for optical coherence tomography,"Opt. Express, 14, 3225 - 3237, 2006.

[12] B. R. Biedermann, W. Wieser, C. M. Eigenwillig, T. Klein, R. Huber, "Dispersion, coherence and noise of Fourier domain mode locked lasers," Opt. Express, 17, 9947 - 9961, 2009. 